\title{
NATIONAL SURVEY ON LABORATORY PRACTICES INRHEUMATOLOGY - TECHNICAL ASPECTS, READING, INTERPRETATION, RELEASE OF ANA REPORTS.
}

Wilton Ferreira Silva Santos ${ }^{1, \star}$, Luís Eduardo Coelho Andrade ${ }^{1}$, Fabiano de Almeida Brito ${ }^{1}$, Leandro Kegler Nardes², Antônio Sérgio Macedo Fonseca ${ }^{1}$, Henrique Luiz Staub ${ }^{1}$, Paulo Louzada Júnior ${ }^{1}$, Max Victor Carioca ${ }^{1}$

1.Comissão de Laboratório da Sociedade Brasileira de Reumatologia, Brazil; 2.Escola Superior de Ciências da Saúde, Brasília (DF), Brazil. *Corresponding author: wiltonss@uol.com.br

\section{BACKGROUND}

Antinuclear antibody (ANA) testing is the serological hallmark of systemic inflammatory rheumatic diseases. Technical procedures in ANA testing have been object of several national and international consensus meetings. The degree to which the various services responsible for training specialists in rheumatology are assimilating the recommendations proposed by these consensus needs to be assessed. The objective was to evaluate the degree to which rheumatology services are adhering to the national and international recommendations on ANA testing.

\section{METHODS}

This is a cross-sectional, exploratory and descriptive study where a structured questionnaire was sent to 52 services that offer a medical residency program in rheumatology in Brazil. The questionnaire consisted of 20 questions focusing on technical aspects of reading, interpreting and releasing the results of ANA testing. Questionnaire was mailed in August of 2019 and answers were tallied up to June of 2020.

\section{RESULTS}

The questionnaire was answered by 41 (78.8\%) of the 52 services contacted. Regarding laboratory practices related to ANA testing, it was observed that $82.9 \%$ adopt the Brazilian Consensus on Autoantibodies (BCA) recommendations, but only 18 services (43.9\%) fully apply these recommendations. The International Consensus on ANA patterns (ICAP) guidelines are followed by 16 services (39.0\%). Among the commercial kits for performing the ANA tests mentioned by the participants, the most used were Euroimmun (36.8\%), Bion (26.3\%) and Inova Diagnostics (21.0\%). The screening dilution adopted by the majority ( $81.6 \%$ ) was $1 / 80$. Thirty-nine percent dilute the reagent samples up to $1 / 640$, and only $15.2 \%$ of the services dilute the sample until final titer is obtained. Mixed/ composite patterns, nuclear reticular coarse speckled and nuclear quasi-homogeneous are recognized by $80.5 \%, 63.4 \%$ and $58.5 \%$ of the participants, respectively. Distinction among the nucleolar patterns is usually made by $65.9 \%$ of the services. Antinuclear antibody exams with isolated cytoplasmic fluorescence are released as reagents by $65.9 \%$, and mitotic patterns are recognized by $73.2 \%$. Clinical correlations are included in the reports by $70.7 \%$ of the participants and $41.5 \%$ adhere to an external quality control program. The nomenclature "ANA - Antinuclear Factor" is still adopted as the name of the exam by $46.3 \%$ of the participants.

\section{CONCLUSION}

The BCA recommendations are adopted by the great majority of the services involved in the training of specialists in rheumatology in Brazil. As less than half of the participants fully follow the BCA recommendations, variability in some aspects of ANA report between services is expected, such as the nonincorporation of certain fluorescence patterns, the nonrecognition of isolated cytoplasmic patterns by $1 / 3$ of the services, and the use of different final dilutions to determine the sample titer.

\section{KEYWORDS}

Antinuclear antibodies, Autoantibodies, Clinical laboratory, Diagnostic test, Autoimmunity. 